Annuaire suisse de politique de développement

27-2 | 2008

Migration et développement: un mariage arrangé

\title{
Les activités transnationales des associations de migrants serbes et kosovars en Suisse
}

Janine Dahinden et Joëlle Moret

\section{(2) OpenEdition}

12 Journals

Édition électronique

URL : http://journals.openedition.org/aspd/650

DOI : 10.4000/aspd.650

ISSN : 1663-9669

Éditeur

Institut de hautes études internationales et du développement

Édition imprimée

Date de publication : 1 décembre 2008

Pagination : 227-238

ISBN : 978-2-940415-07-6

ISSN : 1660-5934

\section{Référence électronique}

Janine Dahinden et Joëlle Moret, « Les activités transnationales des associations de migrants serbes et kosovars en Suisse ", Annuaire suisse de politique de développement [En ligne], 27-2 | 2008, mis en ligne le 01 octobre 2010, consulté le 08 septembre 2020. URL : http://journals.openedition.org/aspd/ 650 ; DOI : https://doi.org/10.4000/aspd.650 


\title{
Les activités transnationales des associations de migrants serbes et kosovars en Suisse
}

\author{
Janine Dahinden et Joëlle Moret*
}

\begin{abstract}
"Being Italian in Italy, Sicilian in Sicily, or Basque in the Pyrenees obviously represents much weaker self-and external identifiers than being Italian in Toronto, Sicilian in Milan or Basque in Montevideo. ${ }^{1}$
\end{abstract}

\footnotetext{
L
}

a migration a pour effet de renforcer les identités collectives fondées sur des critères nationaux, ethniques, religieux ou régionaux. On voit cette lapalissade se concrétiser - sous la forme d'organisations d'immigrés dans toutes les régions du monde qui connaissent des afflux de personnes venues d'ailleurs. Cette «manie de l'association» typique des migrants a suscité de nombreuses études scientifiques, notamment en relation avec l'immigration du début du siècle passé aux Etats-Unis ou celle de l'après-guerre en Suisse. Le caractère frappant du phénomène en question a d'ailleurs donné lieu à quantité de proverbes. On dit par exemple, à propos des immigrés allemands aux EtatsUnis: "Put three Germans together and in five minutes you'll have four clubs», ou sur les Japonais au Brésil: "Two Japanese make an association, and three found a newspaper. $»^{2}$

Les organisations de migrants ${ }^{3}$ sont très diverses à bien des égards, selon le but qu'elles poursuivent, leur degré de formalisation, d'organisation ou de professionnalisme, leur composition et leurs activités, leurs liens avec des institutions locales dans le pays d'accueil ou d'origine. Il y a des organisations homogènes ou hétérogènes quant à l'origine de leurs membres, des associations sportives, des groupes religieux, des associations féminines ou culturelles, des organisations parentales, estudiantines ou professionnelles, ou encore des hometown associations - il est pratiquement impossible d'en faire une liste exhaustive ${ }^{4}$.

* Janine Dahinden, professeure en études sociales et transnationales, Maison d'analyse des processus sociaux (MAPS), Université de Neuchâtel.

Joëlle Moret, collaboratrice scientifique et doctorante, Maison d'analyse des processus sociaux (MAPS), Université de Neuchâtel.

1 Jose C. Moya, «Immigrations and Associations: A Global and Historical Perspective», Journal of Ethnic and Migration Studies, vol. 31, n 5, 2005, p. 839.

2 Ibid., pp. 837-838.

3 Les termes «organisations de migrants» ou «associations de migrants» sont utilisés indifféremment pour désigner les diverses formes possibles de groupement de personnes directement impliquées (première et deuxième génération), non seulement dans le cadre d'associations reconnues officiellement mais aussi dans des structures à caractère plus informel.

4 Pour une typologie, voir par exemple Uwe Hunger, «Ausländervereine in Deutschland. Eine Gesamterfassung auf der Basis des Bundesausländerregisters», in Karin Weiss und Dietrich Thränhardt (Hrsg.), SelbstHilfe. Wie Migranten Netze knüpfen und Sozialkapital schaffen, Freiburg im Breisgau, Lambertus, 2005, pp. 221-244. 
Citons par exemple le FC Kosova qui joue en troisième ligue de l'Association suisse de football, l'association culturelle serbe de Dübendorf qui organise des cours de langue et de danse pour les enfants des immigrés de première génération, le groupe de femmes serbes qui se réunissent tous les mercredis aprèsmidi, et un parti politique albanais qui garde des liens étroits avec sa centrale au Kosovo.

Le seul point commun de ces exemples est qu'il s'agit d'acteurs appartenant tous à la société civile. Ils sont pour le reste parfaitement hétérogènes quant au profil de leurs membres (hommes, femmes, niveau de formation), quant à leurs objectifs et à l'intensité de leur orientation transnationale. On parle de société civile - avec des connotations différentes selon la discipline scientifique intéressée par cette problématique - pour désigner la partie du tissu social qui se développe plus ou moins à l'écart des instances publiques, par des initiatives autonomes des citoyennes et citoyens et de leurs groupements. Ces liens et ces adhésions revêtent une grande importance pour l'intégration d'une société, car ils favorisent une participation active et donnent des repères. Les organisations d'immigrants sont elles-mêmes des acteurs de la société civile. Elles procèdent $\mathrm{du}$ bon vouloir de leurs membres et dépassent le cadre familial ${ }^{5}$. On peut en donner la définition très générale suivante: "Il s'agit de liens sociaux formels ou informels de personnes migrantes à l'intérieur d'une unité territoriale déterminée, liens fondés sur le libre arbitre et motivés par des buts communs allant au-delà d'intérêts purement privés, visant l'autonomie dans les domaines économique et socioculturel. ${ }^{6}$

L'opinion publique admet désormais que les organisations de migrants constituent un facteur important de la dynamique «migration et développement ${ }^{7}$. Ce changement d'éclairage est dû à l'adoption par les sciences sociales d'une perspective transnationale en lieu et place de la priorité donnée jusqu'alors à l'assimilation $^{8}$. Les personnes migrantes construisent une multiplicité d'identités et de réseaux par-delà les frontières, de sorte que leurs organisations adoptent des stratégies elles-mêmes duales et transnationales: elles maintiennent d'une part des liens avec les institutions, les réseaux et les habitants du pays d'origine, d'où une «orientation patriotique» transnationale et, d'autre part, elles cherchent simultanément à participer à la société du pays d'accueil, ce qui correspond à une «orientation locale» à des fins d'intégration. Leurs activités se rapportent en partie au pays d'accueil - par exemple lorsqu'il s'agit d'organiser des manifestations culturelles ou de travailler à l'intégration des migrants. Parallèlement, ces collectivités se préoccupent de ce qui se passe dans leur pays d'ori-

5 Meindert Fennema and Jean Tillie, «Civic Community, Political Participation and Political Trust of Ethnic Groups », Connections, vol. 23, n² 2, 2000, pp. 44-59.

6 Karin Weiss und Dietrich Thränhardt, «Selbsthilfe, Netzwerke und soziales Kapital in der pluralistischen Gesellschaft», in Karin Weiss und Dietrich Thränhardt (Hrsg.), op. cit., pp. 8-44 (NDT: notre traduction).

7 Manuel Orozco and Rebecca Rouse, «Migrant Hometown Associations and Opportunities for Development: A Global Perspective», Migration Information Source, February 2007.

8 Nina Glick Schiller, Linda Green Basch and Cristina Blanc-Szanton, «Transnationalism : A New Analytic Framework for Understanding Migration», in Nina Glick Schiller, Linda Basch and Christina Blanc-Szanton (eds.), Towards a Transnational Perspective on Migration: Race, Class, Ethnicity and Nationalism Reconsidered, New York, New York Academy of Science, 1992, pp. 1-24. Steven Vertovec, «Conceiving and Researching Transnationalism», Ethnic and Racial Studies, vol. 22, n 2, 1999 , pp. 447-462. 
gine: elles organisent des manifestations destinées à faire connaître leurs choix politiques et font du lobbying; elles effectuent des collectes pour satisfaire des besoins d'infrastructure, par exemple dans le domaine des routes ou pour la construction d'écoles dans le pays d'origine. Cette question de l'orientation ne doit en aucun cas déboucher sur un modèle qui serait le pendant des anciens modèles d'assimilation ${ }^{9}$ et dans lequel l'évolution irait nécessairement dans la direction d'une orientation des immigrés axée de façon claire et exclusive sur la Suisse. Il convient plutôt d'envisager une orientation alternée - simultanément ou successivement - entre intégration et préoccupations transnationales.

Le présent article passe en revue les occasions ou les circonstances qui favorisent l'orientation transnationale des organisations de migrants, et examine comment une telle orientation pourrait contribuer au développement de leur pays d'origine. Il traite son sujet en s'appuyant sur un exemple concret, à savoir l'évolution des organisations de Serbes et de Kosovars en Suisse au cours des dernières décennies, ainsi que celle de leurs activités transnationales. Il examine en outre quel rôle ces organisations pourraient jouer dans le développement de leur pays d'origine.

\section{L'évolution des organisations de migrants serbes et kosovars en Suisse}

L'examen de l'évolution en Suisse des organisations de Serbes et d'Albanais du Kosovo depuis 1960 fait apparaître trois phases successives.

\section{Phase 1 (1960-1980): associations "yougoslaves" au service de l'idéologie socialiste}

Les premières associations yougoslaves en Suisse ont vu le jour dans les années 1960, soit à peu près au début du mouvement d'émigration économique vécu par ce pays. Comme d'autres Etats socialistes des Balkans, d'Europe de l'Est et de l'Union soviétique, la Yougoslavie a été un pays fermé durant les premières années de son existence (1945-1964). Il a fallu la pression du chômage provoqué par la profonde réforme économique de 1965 pour que la Yougoslavie commence à ouvrir ses frontières et à légaliser l'émigration $^{10}$. Il faut relever ici que le gouvernement yougoslave justifiait sa politique de migration sur le plan idéologique et social par deux postulats qui - comme nous le verrons - ont également eu leur influence sur la création des associations de migrants en Suisse. Le premier postulat était qu'il s'agissait d'une émigration temporaire, et le second, que les personnes migrantes continuaient de faire partie intégrante de la classe ouvrière yougoslave ${ }^{11}$. En d'autres termes, on mettait l'accent sur le fait que les travailleurs yougoslaves quittaient leur pays pour un temps limité, et que cette émigration à caractère provisoire était au service du développement économique de leur pays. C’est

9 Milton Gordon, Assimilation in American Life, New York, Oxford University Press, 1964.

10 Carl-Ulrik Schierup, «Former Yugoslavia: Long Waves of International Migration», in R. Cohen (ed.), The Cambridge Survey of World Migration, Cambridge, University Press, 1995.

11 Milan Mesic, «External Migration in the Context of the Post-War Development of Yugoslavia », in John B. Allcock, John J. Horton and Marko Milivojevic (eds.), Yugoslavia in Transition, New York; Oxford, Berg, 1992, pp. 171-198. 
ainsi que le séjour et la position des migrants dans les territoires d'immigration, ainsi que leur retour au pays, étaient considérés non pas comme une affaire privée mais comme celle de la société socialiste yougoslave dans son ensemble. Concrètement, l'Etat yougoslave a instauré une véritable régulation des mouvements migratoires et promulgué toute une série de lois et de dispositions relatives au séjour des migrants à l'étranger: des bureaux de recrutement et de placement ont été créés; l'Etat s'est efforcé de passer avec d'autres pays des conventions relatives aux assurances sociales ${ }^{12}$; parallèlement à leurs fonctions habituelles, les représentants diplomatiques des ambassades ou des consulats avaient pour tâche de «protéger» les migrants yougoslaves; ceux-ci étaient encouragés à adhérer aux clubs et associations de leurs compatriotes et à s'engager socialement; on les considérait comme des agents potentiels de développement ${ }^{13}$.

C'est en 1963 qu'a été fondée à Zurich la première association yougoslave, bientôt suivie d'autres - clubs sportifs, associations culturelles ou professionnelles. Beaucoup de ces groupements de l'ère Tito avaient des liens directs avec l'Etat yougoslave par l'intermédiaire de l'ambassade ou du consulat dans le pays d'accueil ${ }^{14}$. Cela signifie que le gouvernement socialiste yougoslave poursuivait dans les années 1970 et 1980 une politique de développement économique qui visait à faire de la migration internationale un facteur de croissance nationale, et que les organisations de migrants étaient des éléments importants de cette politique. Le gouvernement appuyait leur création tout en veillant à ce qu'elles restent dans son camp idéologique et politique. Cette politique de développement n'était donc pas circonscrite aux frontières du pays d'origine, mais conférait à l'Etat yougoslave un caractère transnational ou «déterritorialisé ${ }^{15}$. Dans cette optique, les personnes migrantes restaient des éléments socio-économiques de la communauté nationale et devaient maintenir des liens transnationaux avec leur patrie. L'idée était que les travailleurs émigrés reviendraient au pays et investiraient leurs économies dans des activités productives - exploitations agricoles ou petites entreprises industrielles -, ce qui aurait pour effet de stimuler localement l'essor économique de la Yougoslavie. Les associations yougoslaves à l'étranger étaient à cet égard des vecteurs importants que le gouvernement socialiste avait tout intérêt à instrumentaliser pour concrétiser cette idée.

12 Une convention de ce type a été conclue avec la Suisse en 1964.

13 Zvonimir Baletic, «International Migration in Modern Economic Development: With Special Reference to Yugoslavia», International Migration Review, vol. 6, $\mathrm{n}^{\circ}$ 4, 1982, pp. 736-756. Mathias Lerch, Janine Dahinden and Philippe Wanner, Mapping Three Balkan Diasporas in Switzerland: Development Financing and the Remittance Market in the Balkans and Switzerland: Identification Phase, Neuchâtel, Swiss Forum for Migration and Population Studies (SFM), 2005. Milan Mesic, op. cit.

14 Benno Baumberger, «Zwischen Vergangenheit und Zukunft. Die Bildung der serbischen Community in den 1990er Jahren », in Peter Niederhäuser und Anita Ulrich (Hrsg.), Fremd in Zürich - fremdes Zürich ? Migration, Kultur und Identität im 19. und 20. Jahrhundert, Zürich, Chronos, 2005, pp. 123-133.

15 Arjun Appadurai, «Disjuncture and Difference in the Global Cultural Economy», in Mike Featherstone (ed.), Theory, Culture and Society, London, Sage, 1990, pp. 295-310. 

Graphique 1: Population résidante des ressortissants de pays de l'ancienne Yougoslavie,
1941-2005

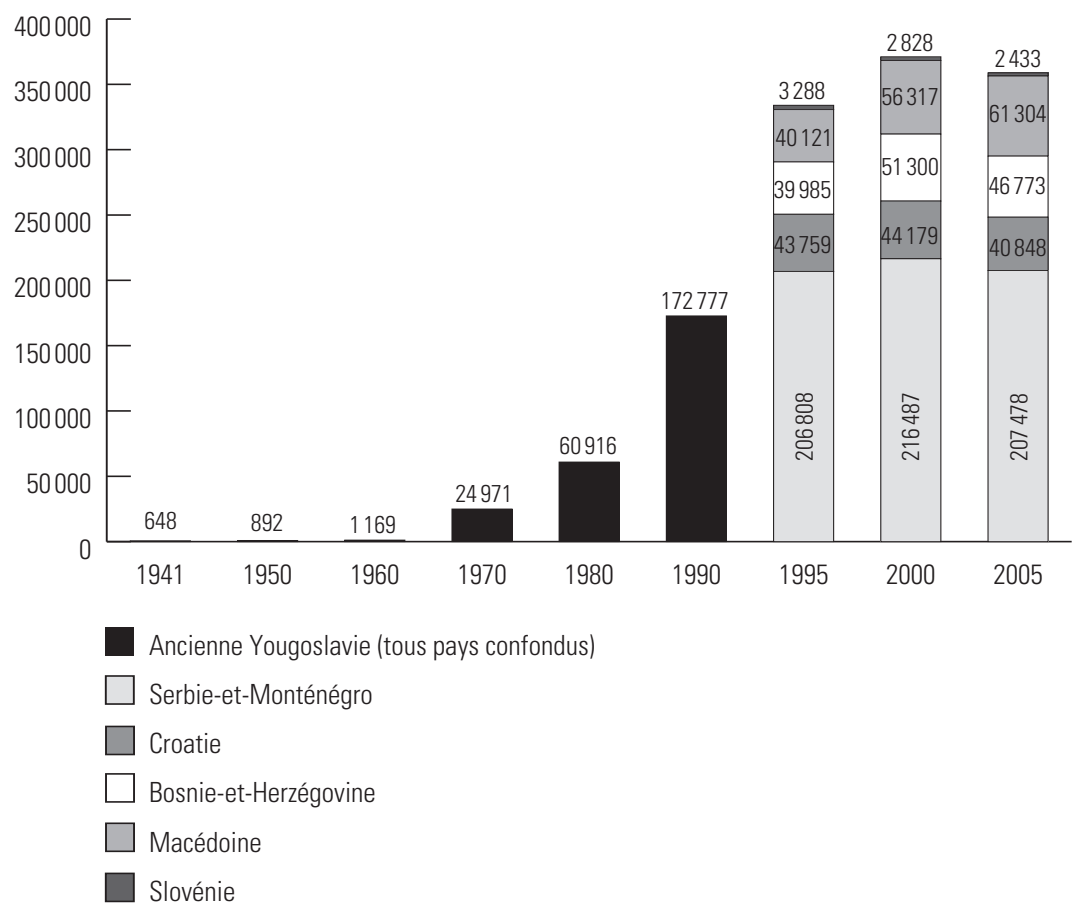

Sources: Office fédéral de la statistique (OFS), Recensement fédéral de la population (jusqu'en 1990) et Statistique de la population résidante de nationalité étrangère (depuis 1995).

Phase 2 (1980-2000):

intensification et autonomie accrue des activités transnationales

Les organisations yougoslaves se sont désagrégées parallèlement aux premiers troubles qui agitaient la Yougoslavie des années 1980, et plus encore lorsque les guerres civiles ont éclaté. Les émigrés se sont alors regroupés selon les nouvelles lignes «ethnonationales», revendiquant leur appartenance identitaire serbe, kosovare, croate, slovène, etc. Il y a ainsi l'exemple de l'association albanaise Perparimi, fondée en 1979 en tant que section de l'association yougoslave (et dans les locaux de celle-ci): à la suite des troubles survenus en 1981 au Kosovo, la section albanaise s'en est détachée pour devenir autonome. Au cours de cette période, les événements de leur région d'origine ont dans l'ensemble beaucoup influencé les activités des organisations de migrants.

La Suisse a alors été le théâtre d'une immigration massive provoquée par la pression des événements survenus dans la région balkanique, et qui prenait la forme du regroupement familial ou de l'asile accordé à des réfugiés ${ }^{16}$. La situation problématique de l'ancienne Yougoslavie incitait les anciens Gastarbeiter

16 Denise Efionayi-Mäder et al., Asyldestination Europa : eine Geographie der Asylbewegungen, Zürich, Seismo, 2001. Etienne Piguet, L'immigration en Suisse depuis 1948. Une analyse des flux migratoires vers la Suisse, Zurich, Seismo, 2005. 
(travailleurs immigrés ou «invités »), désormais au bénéfice d'une autorisation à l'année ou même d'un permis d'établissement (au lieu de l'ancien statut de saisonnier), à faire venir leur famille plutôt qu'à rentrer dans leur pays d'origine ${ }^{17}$. Il en est résulté un bouleversement complet de la structure sociodémographique des Serbes et des Albanais du Kosovo résidant en Suisse: leur nombre a brusquement augmenté, avec une immigration qui s'était fortement féminisée. Les innombrables associations serbes et albanaises du Kosovo qui se sont créées au cours des années 1990 témoignent de cette nouvelle situation démographique. Alors qu'il n'y avait aucune association féminine avant 1990, ces organisations se sont multipliées ensuite parallèlement à l'afflux de femmes venues s'établir en Suisse.

Par ailleurs, la politisation des idées ethniques et nationalistes ne s'est pas limitée au contexte des guerres civiles qui ravageaient l'ancienne Yougoslavie: ces idées étaient à l'époque très présentes aussi chez les personnes de cette région émigrées en Suisse. L'ethnicité devenait une nouvelle catégorie et une langue; elle offrait l'occasion de légitimer les actes et les buts collectifs de divers acteurs, et servait à motiver la création de nouvelles associations ainsi qu'un engagement politique dans les réseaux qui s'étaient constitués. En particulier, les organisations kosovares étaient abondamment fréquentées par des réfugiés reconnus, des personnes qui avaient été obligées de quitter le Kosovo après s'être engagées pour la cause «nationale» et qui bénéficiaient de l'asile en Suisse. Les travailleurs migrants fréquentaient également ces associations, mais leur intérêt tiédissait souvent aussitôt que leur famille les avait rejoints; 1'association avait ici une fonction d'ersatz de la famille ${ }^{18}$.

Beaucoup de ces organisations se sont alors politisées, du côté serbe aussi bien que du côté kosovar. Ce contexte a donné un relief tout particulier à l'élément transnational du phénomène. Certains partis ou groupements politiques du pays d'origine entretenaient des liens directs avec les associations correspondantes établies en Suisse. Deux exemples : 1'organisation estudiantine OTOPOR à Belgrade, qui combattait le régime de Slobodan Milosevic, avait l'appui de plusieurs associations serbes en Suisse, lesquelles mettaient sur pied par exemple un soutien logistique sous forme de tee-shirts, d'appareils de photo, etc., envoyés à Belgrade; pour ce qui est des Albanais du Kosovo, une partie de la Ligue démocratique du Kosovo (LDK) d'Ibrahim Rugova a séjourné un certain temps en Suisse, et ces responsables politiques ont utilisé les réseaux des organisations de migrants pour leurs activités entre la Suisse et le Kosovo ${ }^{19}$.

L'état de guerre dans la région d'origine a par ailleurs exercé une autre influence sur les organisations de migrants en Suisse, dont l'aide humanitaire est alors devenue l'activité principale: ces associations achetaient médicaments, draps, couvertures et autres biens de première nécessité, organisaient elles-

17 Alain Maillard et Ueli Leuenberger, Les damnés du troisième cercle: les Kosovars en Suisse, 1965 1999, Genève, Editions Metropolis, 1999. Hans-Peter von Aarburg, «L'émigration albanaise du Kosovo vers la Suisse. L'imprévisible évolution des projets migratoires», Ethnologie française, vol. 32, n² 2, 2002, pp. 271-282.

18 Janine Dahinden, «Contesting Transnationalism? Lessons from the Study of Albanian Migration Networks from Former Yugoslavia», Global Networks: A Journal of Transnational Affairs, vol. 5, $\mathrm{n}^{\circ} 2$, 2005, pp. 191-208.

19 Mathias Lerch, Janine Dahinden and Philippe Wanner, op. cit. 
mêmes l'acheminement de ces biens vers la Serbie ou le Kosovo et s'occupaient parfois de leur distribution. Les associations de villes d'origine (hometown associations) y participaient souvent, car la politique de recrutement spécifique pratiquée par la Suisse dans les années 1970 et 1980 avait pour effet, entre autres, de susciter une forme particulière de migration en chaîne: les personnes venant d'une région déterminée s'établissaient dans la même localité suisse du fait qu'elles s'étaient mutuellement procuré du travail. C'est ainsi par exemple que les Albanais de Viti ou de Gjilan séjournent presque tous en Suisse romande. Ces migrants se regroupaient alors pour envoyer du matériel de secours dans «leurs» localités d'origine ${ }^{20}$.

En d'autres termes, les guerres civiles qui frappaient la patrie de personnes migrantes suscitaient chez celles-ci un sentiment intense de solidarité, ellemême basée sur l'idée d'une communauté de destin ethnonationale, d'une bounded solidarity ${ }^{21}$ très mobilisatrice. Les organisations de la société civile devenaient ainsi des acteurs importants pour la canalisation et l'organisation logistique des dons philanthropiques, même si leur engagement était souvent teinté de nationalisme.

Cette participation d'organisations privées ne se limite pas à la dimension transnationale de nature politique, mais comporte des aspects économiques. Au cours des années 1990, quelques associations albanaises ont élaboré des projets d'apport de fonds collectifs: des Kosovars de la région de Schaffhouse ont ainsi lancé l'idée de collecter de l'argent (1 franc par jour) auprès de tous les Albanais travaillant en Suisse. Ces ressources financières étaient investies à des fins humanitaires au Kosovo. Quelques années plus tard, c'est la naissance du projet «3\% du salaire», devenu célèbre depuis lors: il était désormais considéré comme normal que chaque Albanais ou Albanaise en Suisse verse 3\% de son salaire pour l'aide au Kosovo. Cet argent était géré par les structures de la LDK, qui en assurait également la distribution. Une partie de cette collecte est allée par exemple à la mise en place d'un système albanais de scolarisation parallèle au Kosovo.

Il convient de relever cependant que les immigrants de l'époque n'adhéraient pas tous à ces organisations. Des études sur le sujet montrent par ailleurs que les activités de ces associations sont (presque) toujours «genrées»: les hommes et les femmes y occupent des fonctions généralement différenciées ${ }^{22}$. Cette remarque s'applique bien à l'exemple évoqué ici: les femmes étaient très présentes dans le domaine humanitaire, tandis que les hommes s'occupaient des affaires politiques - une répartition qui ressemble assez à ce que l'on observe dans nombre d'associations purement helvétiques ${ }^{23}$.

Mais ces réseaux pouvaient bien entendu avoir un double visage: remarquons à cet égard que leurs activités transnationales - politiques ou économiques - sortaient parfois des limites de la légalité ou de la légitimité. On sait par exemple

20 Ibid.

21 Alejandro Portes, «Social Capital: Its Origins and Applications in Modern Sociology», Annual Review of Sociology, vol. 24, $\mathrm{n}^{\circ}$ 1, 1998, pp. 1-24.

22 Marlou Schrover und Floris Vermeulen, «Immigrant Organisations », Journal of Ethnic and Migration Studies, vol. 31, n 5, 2005, pp. 823-832.

23 Janine Dahinden, Prishtina - Schlieren. Albanische Migrationsnetzwerke im transnationalen Raum, Zürich, Seismo, 2005. 
que de l'argent versé par les migrants a financé la lutte armée de l'Armée de libération du Kosovo (UÇK) et que les armes ainsi achetées ont également circulé à travers ces réseaux. Il arrivait aussi que certaines organisations mettent pratiquement «leurs» migrants dans l'obligation de verser une contribution financière.

On constate en résumé que c'est dans la période des plus graves crises serbes et kosovares que les associations créées en Suisse par des ressortissants de ces pays se sont montrées le plus actives. Ce n'était plus l'Etat qui cherchait à instrumentaliser «ses» émigrants: ceux-ci utilisaient leurs propres associations pour avoir eux-mêmes un impact politique et agir dans leur patrie, organiser des actions philanthropiques, mettre sur pied des projets collectifs dans le but d'aider leur coin de pays - un phénomène que l'universitaire américain Benedict Anderson qualifie de «nationalisme à longue distance» (long-distance nationalism). L'ethnicité devenait dans ce contexte une source de motivation: la période en question a ainsi vu naître une communauté de destin caractérisée par des lignes de démarcation ethniques propices à un sentiment puissant de solidarité, lequel a suscité la mobilisation transnationale des migrants qui appartenaient à ces groupes ${ }^{24}$.

\section{Phase 3 (depuis 2000): dissolution, réorientation, nouveaux acteurs}

Un nouveau phénomène se manifeste depuis 2002 environ, plus précisément à partir du moment où la région entame ses processus de pacification et de reconstruction: le nombre d'organisations de la société civile formées de Serbes et de Kosovars en Suisse diminue régulièrement, et les effectifs de celles qui restent sont eux-mêmes en chute libre. En d'autres termes, les activités collectives à caractère transnational d'associations serbes et albanaises du Kosovo sont devenues rares. Quelles explications donner à ce phénomène?

Il semble au premier abord que ces associations aient perdu leur «raison d'être» avec la fin des conflits ouverts dans le pays d'origine. Des objectifs communs tels que la lutte contre le régime de Slobodan Milosevic ou l'organisation de l'aide humanitaire ne sont plus d'actualité. Ou bien une majeure partie des personnes politiquement actives - notamment des Albanais du Kosovo - sont rentrées au pays, laissant à cet égard un vide derrière elles. Bien des migrants serbes et kosovars actuellement en Suisse laissent entendre (parfois avec regret) que l'esprit collectif qui avait marqué les années 1990 a désormais cédé la place à l'individualisme et aux préoccupations familiales. En d'autres termes, la bounded solidarity des années 1990 s'est évaporée.

On observe en même temps que les immigrés serbes, en particulier, ont été déçus par leur patrie. Ils lui reprochent un régime peu démocratique, l'instabilité économique, le clientélisme et la corruption, qui les conduisent à penser qu'un engagement de leur part - de nature économique par exemple - serait peu intéressant et trop risqué ${ }^{25}$. Cela renvoie à un point important, déjà évoqué par Thomas

24 Janine Dahinden, «Deconstructing Mythological Foundations of Ethnic Identities and Ethnic Group Formation: Albanian-speaking and New Armenian Immigrants in Switzerland», Journal of Ethnic and Migration Studies, vol. 34, $\mathrm{n}^{\circ}$ 1, 2008, pp. 55-76.

25 Mathias Lerch, Janine Dahinden and Philippe Wanner, Remittance Behaviour of Serbian Migrants living in Switzerland, SFM-Studies, $n^{\circ}$ 51, Neuchâtel, Swiss Forum for Migration and Population Studies (SFM), 2007. 
Faist $^{26}$, à savoir que les organisations de migrants sont souvent en conflit avec des instances de leur pays d'origine, ou tout au moins en désaccord avec la politique nationale, la bureaucratie, les normes et les valeurs des acteurs locaux. Cette situation fait obstacle à un engagement suivi des migrants ou de leurs associations et freine considérablement les activités transnationales ${ }^{27}$.

Par ailleurs, les organisations serbes et kosovares qui ont subsisté se sont mises à réorienter leurs activités. Elles n'ont plus autant les yeux tournés vers leur patrie, mais s'intéressent au contraire toujours davantage à l'intégration de leurs compatriotes en Suisse. Il y a de nombreuses raisons à ce changement d'orientation: on sait d'une part que les migrants serbes, et plus encore ceux du Kosovo, se caractérisent en général par un statut socio-économique modeste et par un certain degré de marginalisation ${ }^{28}$. Ils sont en même temps frappés de discrimination, par exemple sur le marché de l'emploi ${ }^{29}$. Dans cette optique, il est judicieux que ces associations se concentrent sur les problèmes d'intégration, d'autant plus que les personnes et les familles de ces régions immigrées en Suisse ont renoncé pour le moment à envisager un retour dans leur pays d'origine. Elles se voient conforter dans cette réorientation par le fait que différentes instances publiques - notamment la Commission fédérale pour les questions de migration ou l'Office fédéral des migrations - ont explicitement commencé à impliquer des organisations de migrants dans leur travail de prévention et d'intégration. Comme dans d'autres pays européens, les associations de migrants en Suisse ont depuis quelques années la possibilité d'obtenir des fonds destinés à améliorer l'intégration de leurs compatriotes et à fournir des prestations dans ce sens $^{30}$. La constellation qui s'est dessinée ces dernières années favorise ainsi une orientation vers l'accueil sur le lieu de séjour, tandis que les opportunités structurelles d'une orientation transnationale sont allées en diminuant.

Notre réflexion s'est concentrée jusqu'ici sur les migrants de la première génération. Il convient maintenant d'examiner brièvement ce qu'il en est de la deuxième génération des familles serbes et kosovares, laquelle commence aussi à s'organiser, mais avec des structures et des objectifs autres que ceux des associations traditionnelles de migrants formées par leurs parents. Certains membres de cette deuxième génération s'associent pour transmettre à leurs compatriotes les connaissances et les compétences acquises dans le pays d'accueil (brain transfer). Ces nouvelles idées se concrétisent notamment dans le cadre d'organisations estudiantines. De jeunes Albanaises et Albanais jouissant d'une formation supérieure s'emploient par exemple à venir en aide

26 Thomas Faist, «Migrants as Transnational Development Agents: An Inquiry into the Newest Round on the Migration-Development Nexus », Population, Space and Place, vol. 14, 2007, pp. 21-42.

27 Il sera intéressant à cet égard d'observer ce qui va se passer au Kosovo maintenant qu'il a acquis son indépendance, et quelles seront les réactions des associations de migrants.

28 Philippe Wanner, Migration et intégration. Population étrangères en Suisse. Recensement fédéral de la population 2000, Neuchâtel, Office fédéral de la statistique, 2004.

29 Rosita Fibbi, Bülent Kaya et Etienne Piguet, Le passeport ou le diplôme? Etude des discriminations à l'embauche des jeunes issus de la migration, Rapport de recherche, $\mathrm{n}^{\mathrm{o}} 31$, Neuchâtel, Forum suisse pour l'étude des migrations et de la population (SFM), 2003.

30 Pour un examen critique de ces «politiques de médiation ethnique» entre pouvoirs publics et associations de migrants, cf. Joëlle Moret, Dominik Weber und Janine Dahinden, Gesundheits- und Integrationspolitik mit Netzwerken von MigrantInnen. Arbeitsbericht: "Best practices» und Idealtypen in der Zusammenarbeit mit Netzwerken von MigrantInnen, Neuenburg, Schweizerisches Forum für Migrations- und Bevölkerungsstudien (SFM), 2007. 
à des compatriotes défavorisés, entre autres pour trouver un emploi; c'est en quelque sorte un «projet de mentorat». Autre exemple: un groupe de jeunes Kosovars fait profiter de ses compétences des compatriotes désireux de créer une entreprise dans leur région d'origine ${ }^{31}$. Ces exemples appellent toutefois une certaine réserve en ce sens qu'ils restent marginaux et s'expriment sous forme d'idées plutôt que par des actes concrets.

\section{Pour conclure, quelques réflexions sur les facteurs favorisant les activités transnationales}

Le présent article avait pour but de donner une idée du rôle des organisations de migrants eu égard à la problématique «migration et développement». Nous avons décrit à titre d'exemple l'évolution des associations de migrants serbes et kosovars en Suisse, dont il est possible de tirer quelques conclusions prudentes.

Il est manifeste que certaines constellations structurelles peuvent favoriser ou freiner un engagement transnational des migrants. Au niveau des autorités et des institutions publiques, les événements et les acteurs du pays d'origine, aussi bien que ceux du pays d'accueil, exercent une influence déterminante sur la structure et les activités des organisations de migrants. Ils agissent également sur l'orientation plus ou moins transnationale de ces activités - comme d'autres études l'ont déjà établi ${ }^{32}$. Un gouvernement fort dans le pays d'origine - par exemple le régime socialiste à l'époque de Tito - peut instrumentaliser des associations situées à l'étranger et renforcer leur caractère transnational. Il en va de même pour la politique d'intégration dans le pays d'accueil: si cette politique aide financièrement les organisations de migrants dans leurs activités d'intégration, cela tend à les orienter davantage sur le pays où elles se trouvent. On constate ainsi que les pouvoirs publics créent des structures agissantes sur l'orientation bipolaire de ces associations (avec un pôle local et un pôle transnational).

De même, le contexte du pays d'accueil et celui du pays d'origine revêtent l'un et l'autre une très grande importance pour l'orientation de ces organisations de la société civile: non seulement le nationalisme et la politique ethnique de la région d'origine conduisaient les associations à adopter de nouvelles lignes - et la Suisse à osciller entre accueil et rejet des immigrants -, mais ils tendaient à multiplier le nombre de groupements et à intensifier leur engagement transnational. Cette période de guerres et de crises dans leur région d'origine a suscité des projets philanthropiques de diverses couleurs politiques sous le patronage des organisations d'immigrés.

Mais l'orientation plus ou moins transnationale d'une association dépend aussi des caractéristiques du groupe d'immigrés, comme sa composition démographique (par exemple une association de femmes immigrées), son statut socioéconomique ou le type de permis de séjour de ses membres; on peut ainsi se demander dans quelle mesure les immigrés de la première génération provenant de ces pays d'origine disposent des ressources (capital culturel, économique et

31 Mathias Lerch, Janine Dahinden und Philippe Wanner, Mapping Three Balkan Diasporas in Switzerland, op. cit.

32 Patricia Landolt, «The Transnational Geography of Immigrant Politics: Insights from a Comparative Study of Migrant Grassroot Organising», The Sociological Quarterly, n 49, 2008, pp. 53-77. 
social $)^{33}$ nécessaires pour devenir concrètement des agents de développement, car ce sont des groupes fortement marginalisés et en partie discriminés dans le contexte suisse. Cela pourrait expliquer en partie, outre les autres raisons évoquées, pourquoi l'orientation transnationale des associations d'immigrés est devenue hésitante depuis la fin de la guerre. Réunir du matériel de secours ne demande pas les mêmes ressources qu'alimenter durablement des projets d'infrastructure dans le pays d'origine - surtout lorsqu'il s'agit de projets qui dépassent le cadre familial et exigent de gros investissements. Le contexte de l'accueil est primordial à cet égard: il offre, en matière de capital et de ressources, certaines opportunités indispensables pour mettre en place un engagement transnational durable. Et c'est ici que la deuxième génération peut avoir un impact particulier: elle élaborera peut-être une nouvelle manière d'établir des relations transnationales, dans une perspective que l'on peut espérer durable en raison de ressources bien plus substantielles - entre autres sur le plan de la formation que celles de la première génération.

En tout état de cause, l'exemple choisi ici ne saurait être généralisé sans réserve. Nous sommes par conséquent d'avis qu'il vaudrait la peine d'entreprendre d'autres études afin d'approfondir ce sujet de manière systématique.

\section{Bibliographie}

Aarburg, Hans-Peter von, «L'émigration albanaise du Kosovo vers la Suisse. L'imprévisible évolution des projets migratoires », Ethnologie française, vol. 32, n 2, 2002, pp. 271-282.

Appadurai, Arjun, «Disjuncture and Difference in the Global Cultural Economy», in Mike Featherstone (ed.), Theory, Culture and Society, London, Sage, 1990, pp. 295-310.

Baletic, Zvonimir, «International Migration in Modern Economic Development: With Special Reference to Yugoslavia», International Migration Review, vol. 6, n 4, 1982, pp. 736-756.

Baumberger, Benno, «Zwischen Vergangenheit und Zukunft. Die Bildung der serbischen Community in den 1990er Jahren », in Peter Niederhäuser und Anita Ulrich (Hrsg.), Fremd in Zürich - fremdes Zürich? Migration, Kultur und Identität im 19. und 20. Jahrhundert, Zürich, Chronos, 2005, pp. 123-133.

Bourdieu, Pierre, «Ökonomisches Kapital, kulturelles Kapital, soziales Kapital», in Reinhard Kreckel (Hrsg.), Soziale Ungleichheiten, Soziale Welt, Sonderband 2, Göttingen, Schwarz, 1983, pp. 183-198.

Dahinden, Janine, «Contesting Transnationalism? Lessons from the Study of Albanian Migration Networks from former Yugoslavia», Global Networks: A Journal of Transnational Affairs, vol. 5, $\mathrm{n}^{\circ} 2$, 2005, pp. 191-208.

Dahinden, Janine, «Deconstructing Mythological Foundations of Ethnic Identities and Ethnic Group Formation: Albanian-speaking and New Armenian Immigrants in Switzerland», Journal of Ethnic and Migration Studies, vol. 34, nº 1, 2008, pp. 55-76.

Dahinden, Janine, Prishtina - Schlieren. Albanische Migrationsnetzwerke im transnationalen Raum, Zürich, Seismo, 2005.

Efionayi-Mäder, Denise et al., Asyldestination Europa: eine Geographie der Asylbewegungen, Zürich, Seismo, 2001.

Faist, Thomas, «Migrants as Transnational Development Agents : An Inquiry into the Newest Round on the Migration-Development Nexus », Population, Space and Place, vol. 14, 2007, pp. 21-42.

Fennema, Meindert and Jean Tillie, «Civic Community, Political Participation and Political Trust of Ethnic Groups », Connections, vol. 23, n² 2, 2000, pp. 44-59.

Fibbi, Rosita, Kaya Bülent et Etienne Piguet, Le passeport ou le diplôme? Etude des discriminations à l'embauche des jeunes issus de la migration, Rapport de recherche, $\mathrm{n}^{\mathrm{o}} 31$, Neuchâtel, Forum suisse pour l'étude des migrations et de la population (SFM), 2003.

33 Pierre Bourdieu, «Ökonomisches Kapital, kulturelles Kapital, soziales Kapital», in Reinhard Kreckel (Hrsg.), Soziale Ungleichheiten, Soziale Welt, Sonderband 2, Göttingen, Schwarz, 1983, pp. 183-198. 
Gordon, Milton, Assimilation in American Life, New York, Oxford University Press, 1964.

Hunger, Uwe, «Ausländervereine in Deutschland. Eine Gesamterfassung auf der Basis des Bundesausländerregisters », in Karin Weiss und Dietrich Thränhardt (Hrsg.), SelbstHilfe. Wie Migranten Netze knüpfen und Sozialkapital schaffen, Freiburg im Breisgau, Lambertus, 2005, pp. 221-244.

Landolt, Patricia, «The Transnational Geography of Immigrant Politics: Insights from a Comparative Study of Migrant Grassroot Organising », The Sociological Quarterly, n 49, 2008, pp. 53-77.

Lerch, Mathias, Janine Dahinden and Philippe Wanner, Mapping Three Balkan Diasporas in Switzerland: Development Financing and the Remittance Market in the Balkans and Switzerland: Identification Phase, Neuchâtel, Swiss Forum for Migration and Population Studies (SFM), 2005.

Lerch, Mathias, Janine Dahinden and Philippe Wanner, Remittance Behaviour of Serbian Migrants Living in Switzerland, SFM Studies, $\mathrm{n}^{\circ}$ 51, Neuchâtel, Swiss Forum for Migration and Population Studies (SFM), 2007.

Maillard, Alain et Ueli Leuenberger, Les damnés du troisième cercle: les Kosovars en Suisse, 1965-1999, Genève, Editions Metropolis, 1999.

Mesic, Milan, «External Migration in the Context of the Post-War Development of Yugoslavia», in John B. Allcock, John J. Horton and Marko Milivojevic (eds.), Yugoslavia in Transition, New York; Oxford, Berg, 1992, pp. 171-198.

Moret, Joëlle, Dominik Weber und Janine Dahinden, Gesundheits- und Integrationspolitik mit Netzwerken von MigrantInnen. Arbeitsbericht: «Best practices» und Idealtypen in der Zusammenarbeit mit Netzwerken von MigrantInnen, Neuenburg, Schweizerisches Forum für Migrations- und Bevölkerungsstudien (SFM), 2007.

Moya, Jose C., «Immigrations and Associations: A Global and Historical Perspective», Journal of Ethnic and Migration Studies, vol. 31, n 5, 2005, pp. 833-864.

Orozco, Manuel and Rebecca Rouse, «Migrant Hometown Associations and Opportunities for Developement: A Global Perspective», Migration Information Source, February 2007, <http://www. migrationinformation.org/Feature/print.cfm?ID=579>.

Piguet, Etienne, L'immigration en Suisse depuis 1948. Une analyse des flux migratoires vers la Suisse, Zurich, Seismo, 2005.

Portes, Alejandro, «Social Capital: Its Origins and Applications in Modern Sociology », Annual Review of Sociology, vol. 24, nº 1, 1998, pp. 1-24.

Schierup, Carl-Ulrik, «Former Yugoslavia: Long Waves of International Migration», in R. Cohen (ed.), The Cambridge Survey of World Migration, Cambridge, UK, Cambridge University Press, 1995.

Schiller, Nina Glick, Linda Green Basch and Cristina Blanc-Szanton, «Transnationalism: A New Analytic Framework for Understanding Migration», in Nina Glick Schiller, Linda Basch and Christina Blanc-Szanton (eds.), Towards a Transnational Perspective on Migration: Race, Class, Ethnicity and Nationalism Reconsidered, New York, New York Academy of Science, 1992, pp. 1-24.

Schrover, Marlou and Floris Vermeulen, «Immigrant Organisations », Journal of Ethnic and Migration Studies, vol. 31, $\mathrm{n}^{\circ}$ 5, 2005, pp. 823-832.

Vertovec, Steven, «Conceiving and Researching Transnationalism», Ethnic and Racial Studies, vol. 22, $\mathrm{n}^{\circ} 2,1999$, pp. 447-462.

Wanner, Philippe, Migration et intégration. Populations étrangères en Suisse. Recensement fédéral de la population 2000, Neuchâtel, Office fédéral de la statistique, 2004.

Weiss, Karin und Dietrich Thränhardt, «Selbsthilfe, Netzwerke und soziales Kapital in der pluralistischen Gesellschaft», in Karin Weiss und Dietrich Thränhardt (Hrsg.), SelbstHilfe. Wie Migranten Netzwerke knüpfen und soziales Kapital schaffen, Freiburg im Breisgau, Lambertus, 2005, pp. 8-44. 